\title{
Issues of Indeterminism in the Polish School of Logic
}

\author{
Jak Simoni \\ "Aleksandër Moisiu" University Durrës, Albania \\ jaksimoni@yahoo.com
}

\section{Doi:10.5901/jesr.2013.v3n7p151}

\begin{abstract}
Dealing with the history of logic and its relation to philosophy, I have noticed the significant contribution given hereto by the representatives of the Polish School of Logic, and especially of its main representative Jan Łukasieicz. This author has discussed thoroughly indeterminism, which he considers as a metaphysical substrate of the polyvalent logic, introduced by himself. His arguments in favor of indeterminism are taken from everyday life and by exemplifying the absurdity of the opposite theory, i.e. determinism. Through his research he concludes to the polyvalent logic.

Such issues and interpretations of other authors will be discussed in the following article.
\end{abstract}

Keywords: modality, bivalence, determinism, indeterminism.

\section{Introduzione}

Pur non coincidendo all'origine, lo dobbiamo ammettere che lo sviluppo del pensiero logico e filosofico si sono influenzati continuamente tra di loro e la natura del pensiero ha sempre rappresentato il loro punto di coincidenza. Pur essendo più denso all'origine, quando le science erano indistinguibili e non avevano preso le loro strade separate, questo intreccio ha accompagnato tutta la storia della logica e della filosofia raggiungendo l'epoca moderna e contemporanea. Nel campo logico la tradizione aristotelica e di conseguenza la logica bivalente dominarono questo campo del sapere fin al ventesimo secolo. Un evento importante che modificha il corso della storia della logica e anche della filozofia è l'invenzione nei primi decienni del secolo scorso della logica polivalente dal rappresentane più importante della scuola logica polacca, Jan Łukasiewicz. A questa scoperta precede una lunga discussione filosofica sui oggetti logici, sui principi logici e sulla logica di Aristotele. I suoi principi vengono trattati come processi di pensiero e la validità universale si sostituisce con il consenso interindividuale. Risultato di questa lunga discussione è la nuova logica non aristotelica con più valori di verità. La lunga tradizione della logica classica viene messa in discussione. Le modalità vengono accettate e le proposizioni possibili vengono inseriti nella struttura logica strutturando un sistema logico più ricco con una verifica complessa distribuendo di valori di verità tramite matrici.

\section{Oggetti logici e principi logici secondo Twardowski, Meinong e kukasiewicz}

Come rappresentante più importante della scuola polacca, che rappresenta l'argomento della nostra discussione, è il filosofo e logico Jan Łukasiewicz. Per comprendere meglio il suo pensiero trattiamo brevemente anche il pensiero di Twardowski, Meinong sugli oggetti logici. Questi due autori hanno avuto una grande influenza sul pensiero di quest'ultimo. Con Twardowski il legame è più importante. Łukasiewicz si laureò a Leopuli nel 1902 sotto la sua direzione. Invece con Meinong ebbe un contatto diretto a Graz fra 1908 e 1909 in un seminario filosofico tenuto dallo stesso Meinong. La lunga discussione sugli oggetti logici contribuisce nel rivedere la validità dei principi logici classici. Accettando come indiscutibile il principio del terzo escluso i valori di verità di una proposizione non possono essere estesi fuori ai due tradizionali. Questa discussione precederà la logica polivalente.

Per quanto riguarda gli oggetti logici e mentali Twardowski distingue il duplice significato di "rappresentato". Secondo egli, il contenuto è pensato, rappresentato. Ciò che è rappresentato in una rappresentazione è il suo contenuto; ciò che è rappresentato per mezzo di una rappresentazione è il suo oggetto. È chiaro che sono gli oggetti che si rappresentato nella nostra mente. Secondo Twardowski, rappresentazioni e giudizi sono due classi distinti di fenomeni psichici a causa del diverso tipo di riferimento intenzionale all'oggetto e l'esistenza del giudizio consiste nel affermare e nel negare, ed affermare un oggetto A significa affermare l'esistenza di A e le sue parti costituenti, mentre nel negare viene negato l'oggetto A ma, non l'esistenza di tutte le parti costituenti. (Raspa. p.231). Poiché affermare 0 
negare significa asserire l'esistenza o meno dell'oggetto su cui verte il giudizio, il contenuto del giudizio non può che consistere nell'esistenza dell'oggetto di cui si tratta. Quello che in questo caso viene giudicato è l'oggetto stesso.

Twardowski svolge anche un analisi della rappresentazione di "niente" e delle rappresentazioni contraddittorie, di quelle non fattuali. "Niente" non è una rappresentazione perché solo i termini categorematici (greci, non greci) disegnano rappresentazioni. Se si considera bene "niente" è solo componente di proposizioni negative (niente è eterno). Invece nella rappresentazione "quadrato con angoli obliqui" il contenuto correlato a questo atto di rappresentazione costituisce il significato del nome. Questo nome non significa qualcosa ma nomina qualcosa, e precisamente qualcosa che ha proprietà contraddittorie, e cui si nega l'esistenza non appena ci si vede indotti a annunciare un giudizio intorno a ciò che si è nominato. Ma qualcosa è disegnato mediante il nome anche se ciò non esiste. Per Twardowski il contenuto esiste anche nel caso in cui l'oggetto non esiste. In un giudizio negativo vero negare un oggetto implica che esso si rappresenti ed è questo un fatto psichico. Questo fatto succede come conseguenza delle capacità della mente di paragonare. Mentre la rappresentazione è sempre reale l'oggetto a volte si a volte no.

In questo terreno opera anche Meinong che abbiamo ricordato sopra. Per Meinong tutto è "oggetto" non solo le cose reali, i referenti e i significati delle espressioni linguistiche a prescindere dal fatto che abbiano o no dei referenti reali esterni(Raspa. p.242). Secondo Meinong oltre agli oggetti reali che "esistono" si danno anche gli oggetti ideali che "sussistono" ma non esistono. Questi ultimi cadono nel ambito del essere solo in parte. Sussistenti sono oggetti ideali come numeri, relazioni, concetti ecc. Sono al di fori dell'essere gli oggetti che, né sussistono, né esistono e si dividono in possibili, pero privi di essere e impossibili (contradditori come il quadrato che abbia l'area di un cerchio di raggio una unita o diversamente chiamato quadrato rotondo). Meinong distingue fra objekt, l'oggetto della rappresentazione e ojektiv, l'oggetto del giudizio e dell'assunzione. La differenza fra giudizio e assunzione sta nella presenza, nel primo, del momento della convinzione, che manca nella seconda. La possibilità di predicare determinate proprietà su un oggetto, che non esiste, presuppone che in qualche modo viene dato.

Dopo aver visto questi autori che accettano le rappresentazioni del "niente" e degli "oggetti contradditori", allora è impossibile in queste circostanze, non mettere in dubbio i principi logici di non contraddizione e del terzo escluso ritenuti evidenti in tutta la storia della logica ed è questo lo scopo di Łukasiewicz e l'influenza sul suo pensiero dell'interpretazione di questi due autori è notevole. Łukasiewicz nel libro Del principio di contraddizione in Aristotele, dove tramite un analisi dal punto di vista psicologico, ontologico e logico, cerca di dimostrare che il principio di non contraddizione non e sempre valido ed accenna la possibilità di una logica non aristotelica. In questo scritto si discute anche il principio del terzo escluso(Łukasiewicz.1910:95). Anche questo principio, secondo Łukasiewicz, dipende dalla distinzione degli oggetti in completi (gli oggetti reali spazio temporali) e incompleti (oggetti ideali prodotti dallo spirito umano). Una proposizione che verte sui primi è vera o falsa mentre ciò non vale per i secondi, poiché essi non sono sufficientemente determinati. Egli sostiene che il principio del terzo escluso non è un principio vero per se stesso, ne può essere dimostrato, per cui va considerato come una supposizione, ma indispensabile ai fini pratici. Egli afferma che l'applicazione del principio del terzo escluso agli oggetti reali è collegato con il postulato della determinazione universale dei fenomeni, non soltanto quelli passati o presenti, ma anche di quelli futuri. Se qualcuno negasse che tutti i fenomeni futuri siano già predestinati sotto tutti i rispetti, probabilmente egli non accetterebbe il principio in questione.

\section{Per un destino indeterminista dell'uomo.}

La questione del determinismo non è soltanto una questione che serve come sostrato della logica non aristotelica ma una questione collegata direttamente con il destino e l'attività dell'uomo. L'uomo essendo soggetto d'azione usa il sapere non soltanto per scopi teoretici, ma anche pratici. Chi agisce ha da fare con cose che non sono sempre le stesse, ma possono essere anche diverse. In esse, egli scopre gli aspetti su cui ha da agire. II suo sapere deve guidare la sua azione (Gadamer. tr.it.p. 651).

Non escludendo del tutto il pensare teoretico dobbiamo accettare che l'uomo pensa per agire. Piegarsi passivamente al destino oppure mobilitarsi con tutte le forze a modificare il corso degli eventi, è una questione che preoccupa non soltanto ogni studioso di scienza o filosofia, ma ogni uomo. Per evitare gli inconvenienti e trarre più profitto possibile, istintivamente l'uomo, ed ogni essere vivente, cerca di orientarsi nel mondo e di adeguarsi ai fenomeni e agli eventi. Nella complessità degli eventi l'uomo è costretto a scegliere 0 evitare, rifiutare 0 accettare, accontentarsi dello stato di cose o cercare di modificarle. Davanti a queste scelte l'uomo si mette in una dura prova e quindi deve riflettere, ragionare e calcolare le probabilità degli eventi. La capacità di capire e di prevedere il corso degli eventi comporta benefici, l'incapacità comporta rischi. Molti filosofi hanno trattato largamente queste questioni tracciando due linee filosofiche, quella del determinismo e del indeterminismo. Queste questioni sono trattate anche in alcuni momenti 
della storia della logica, soprattutto di quella modale. Un importante contributo ha dato il filosofo che stavamo trattando Jan Łukasiewicz. Questo filosofo ha trattato l'indeterminismo ma collegandolo sempre con i problemi logici perche lo considera come sostrato metafisico della logica polivalente che sta scoprendo. Gli argomenti a favore del indeterminismo gli trova nella storia della filosofia ma anche prendendo semplici esempi della vita quotidiana dimostrando l'assurdità del determinismo. Secondo il determinismo tutto ciò che succede è predestinato ed il corso degli eventi non si può modificare dalla forza della volontà dell'uomo. Dalla storia della filosofia recupera la libera volontà e la capacità di scelta dell'uomo come argomenti contro il determinismo. La tradizione filosofica indeterminista che attribuisce all'uomo la libera volontà è estremamente ricca in tutta la storia della filosofia. II senso indeterministico si distingue chiaramente nel pensiero aristotelico. Nell'opera Della interpretazione Aristotele riteneva che alla contingenza è costitutivamente connessa la scelta, la quale ha luogo soltanto nel dominio di ciò che non è necessario che sia cosi com'è, e nella scelta si specifica l'attività pratica nella sua valenza morale"( tr.it di Zanata M., Milano1992.p. 51). L'uomo deve fare delle scelte che hanno senso soltanto nel dominio di ciò che non è necessario quindi in una realtà contingente. Aristotele lascia $\mathrm{i}$ fenomeni fisici in preda al caso.

Per quando riguarda la libera volontà ci sono stati filosofi che glielo attribuivano anche agli animali. Uno di loro è Pierre Bayle. Secondo egli non c'è ragione di togliere alle bestie la libertà (Bayle. tr.it.324). Łukasiewicz considera il determinismo imprescindibile dalla questione della libera volontà. Secondo egli il determinismo rigetta la libera volontà. Per loro ogni fatto è vero anche nei tempi precedenti e futuri. Tra il passato e il futuro non c'è differenza; l'unica differenza è che il passato è trascorso e il futuro trascorrerà; non c'è differenza dal punto di vista della verità. Noi esistiamo in questo mondo che ci circonda e non conosciamo come finirà. Ogni bene ed ogni male è predeterminato nel passato, ed e predeterminato il momento della morte di ciascuno. Noi siamo soltanto burattini in questa dramma universale e non rimane nient'altro da fare che aspettare tranquillamente la fine!" (Łukasiewicz, Selekted works. p. 113). Inoltre la teoria determinista è strettamente legata anche alla teoria della causa e dell'effetto. Łukasiewicz spiega come i deterministi usano questa teoria a loro favore. Per Łukasiewicz, il fatto che tutti gli eventi siano casualmente determinati non comportano che siano predestinati dall'inizio. ( Ibid.:117).

Łukasiewicz non può accettare questa situazione dove l'uomo viene privato dalla sua libertà di decidere e dalla sua volontà. Secondo egli possiamo decidere, volere e rifiutare, scegliere ed evitare. Per fare ciò bisogna cercare di conoscere i segreti dei fenomeni della natura e del mondo che ci circonda, una impresa questa non facilmente raggiungibile. Pur accentando la complessità della situazione umana, Łukasiewicz richiama il protagonismo dell'uomo. L'uomo non deve sempre subire ma con la forza della volontà e guidato dalla ragione deve tentare di migliorare il proprio destino, ogni tanto anche contrastando ciò che è realmente. L'uomo deve essere libero e responsabile nella scelta del bene 0 del male. Queste questioni vengono trattate largamente e in diversi aspetti con uno scopo che non sembra solamente teoretico ma anche pratico. Ricuperando la libera volontà per quando riguarda le questioni umani Łukasiewicz ricupera il possibile in logica e scopre la logica polivalente.

\section{Una nuova logica non crissipea}

Tutto il dibattito a favore del indeterminismo e degli eventi possibili di cui abbiamo parlato porta all'invenzione della nuova logica che Łukasiewicz chiama non crissipea, perche i stoici si distinguevano come sostenitori della logica bivalente. L'indeterminismo, secondo Łukasiewicz, rappresenta il substrato metafisico della nuova logica non classica. I sostenitori di questa nuova logica sono indeterministi, mentre i suoi oppositori sono deterministi. Per una proposizione che oggi non è né vera né falsa ma e possibile, Łukasiewicz introduce un altro valore di verità che inizialmente la denota con 2 e poi sempre con la frazione 1/2. Introducendo questa possibilità schematizza un sistema logico inizialmente con tre valori di verità e poi un sistema polivalente con infiniti valori di verità. Diventa cosi possibile la costruzione delle tavole di verità inizialmente con il terzo valore introdotto e poi con infiniti valori. La tavola della verità della negazione nel nuovo sistema logico con tre valori di verità diventa :

\begin{tabular}{|c|c|}
\hline $\mathrm{P}$ & $\sim \mathrm{P}$ \\
\hline 0 & 1 \\
$1 / 2$ & $1 / 2$ \\
1 & 0 \\
\hline
\end{tabular}


Invece l'implicazione ha la tavola della verità seguente:

\begin{tabular}{|c|ccc|}
\hline $\mathrm{P} \rightarrow \mathrm{Q}$ & 0 & $1 / 2$ & 1 \\
\hline 0 & 1 & 1 & 1 \\
$1 / 2$ & $1 / 2$ & 1 & 1 \\
1 & 0 & $1 / 2$ & 1 \\
\hline
\end{tabular}

In questa maniera sono costruite le tavole di verità anche per la congiunzione, disgiunzione e l'equivalenza inizialmente con tre valori di verità puoi con infiniti valori di verità.

In fine aggiungiamo che con il grande contributo di Łukasiewicz la simbolica della scuola logica polacca diventa del tutto originale:

è possibile che $p$ in simbolica polacchi si scrive $\mathrm{Mp}$

non è possibile che $p$ in simbolica polacchi si scrive NMp

è possibile che non $p$ in simbolica polacchi si scrive MNp

non è possibile che non $p$ in simboli polacchi si scrive NMNp

dove la lettera ' $p$ ' disegna una qualsiasi proposizione, ' $N$ ' è il simbolo della negazione; ' $M$ ' disegna la possibilità. Non è possibile che non $p$ si può esprimere è necessario che $p$. Invece l'implicazione $p \rightarrow q$ viene disegnata Cpq e la congiunzione $p \wedge q$ viene disegnato $\mathrm{Kpq}$. In questa maniera si costruisce un sistema logico del tutto particolare.

\section{References}

Aristotele, Della interpretazione (tr.it di Zanata M.Rizzoli, Milano1992)

Aristotele, Opere,La terza1992, Roma - Bari 1988

Gadamer H. G, Wahrheit und Methode. J.C.B. Mhor (Paul Siebeck) Tübingen1968

Kneale W.C.e M (tr. It., Storia della logica, Enaudi, Torino 1972.)

Lukasiewicz Jan, Logica modale.Faenza,1979

Lukasiewicz Jan, Selekted works.North-Holland Publiscig-Amsterdam,1970

Lukasiewicz Jan, O zasadzie sprzeczn'osci u Ariystotelesa, 1910 (tr. it. Del principio di contraddizione in Aristotele. Quodlibet, Macerata 2003)

Pierre Bayle, tr. it. Dizionario storico e critico, a cura di Cantelli.G. Laterza 1977

"Paradigmi"XVIII n.53, Roma 2000

Popper Karl, Logik der Forschung, Julius Springer, Wien 1934 (tr.it. Logica della scoperta scientifica, Enaudi, Torino,1970)

Raspa V In contradizione,Edizioni Parnaso, Trieste 1999

Wittgenstein Ludwig, Tractatus logico-philosophicus, Routledge and Kegan Paul, London, 1922 (tr.it. Enaudi, Torino,1964) 